\title{
Theoretical Study of the Multiferroic Behavior of the Magnetic Relaxor Ferroelectric $\mathrm{CdCr}_{2} \mathrm{~S}_{4}$
}

\author{
Angel T. Apostolov1, Iliana N. Apostolova², Julia M. Wesselinowa ${ }^{3^{*}}$ \\ ${ }^{1}$ Department of Physics, Faculty of Hydrotechnics, University of Architecture, Civil Engineering and Geodesy, Sofia, Bulgaria \\ ${ }^{2}$ Faculty of Forest Industry, University of Forestry, Sofia, Bulgaria \\ ${ }^{3}$ Department of Physics, University of Sofia, Sofia, Bulgaria \\ Email: ^julia@phys.uni-sofia.bg
}

How to cite this paper: Apostolov, A.T., Apostolova, I.N. and Wesselinowa, J.M. (2018) Theoretical Study of the Multiferroic Behavior of the Magnetic Relaxor Ferroelectric $\mathrm{CdCr}_{2} \mathrm{~S}_{4}$. Advances in Materials Physics and Chemistry, 8, 459-467. https://doi.org/10.4236/ampc.2018.812031

Received: December 28, 2018

Accepted: January 20, 2019

Published: January 23, 2019

Copyright (c) 2018 by author(s) and Scientific Research Publishing Inc. This work is licensed under the Creative Commons Attribution International License (CC BY 4.0).

http://creativecommons.org/licenses/by/4.0/

\begin{abstract}
Using a microscopic model and the replica method as well as the Green's function theory, we have investigated the relaxor and multiferroic behavior of $\mathrm{CdCr}_{2} \mathrm{~S}_{4}$. The magnetization, the remanent polarization $P_{r}$ and the real part of the dielectric function $\epsilon^{\prime}$ are studied theoretically as a function of temperature, with and without a magnetic field, respectively. The magnetization and the polarization exist together below the magnetic phase transition temperature. $P_{r}$ decreases whereas $\epsilon^{\prime}$ increases and the peak shifts to smaller temperature values with increasing magnetic field $h$. Moreover, the temperature and electric field $E$ dependence of the magnetization $M$ is also discussed. A kink is observed around the ferroelectric transition temperature. The kink is deeper for stronger electric fields and anharmonic spin-phonon interactions.
\end{abstract}

\section{Keywords}

Multiferroic $\mathrm{CdCr}_{2} \mathrm{~S}_{4}$, Microscopic Model, Polarization, Dielectric Function

\section{Introduction}

The magnetic relaxor ferroelectric materials with the coexistence of relaxor ferroelectricity and magnetic order have been attracting considerable attention due to their peculiar properties and various applications [1] [2] [3] [4]. Basic components of this family of materials are the chromium chalcogenide and the compounds that have the general formula $\mathrm{ACr}_{2} \mathrm{X}_{4}$, where $\mathrm{A}=\mathrm{Cd}$ or $\mathrm{Hg}, \mathrm{X}=\mathrm{S}$ or Se. These crystals are generally found in the cubic spinel structure. It is well known that $\mathrm{Cr}^{3+}$ with spin $S=3 / 2$ is responsible for the magnetic properties [5]. As a relaxor ferroelectric, structural investigation indicates that the system is close to a structural instability and there is a possible off-center displacement of 
$\mathrm{Cr}^{3+}$ ions [6] [7]. Such an off-center position, in conjunction with geometrical frustration, leads to a relaxor ferroelectric state [2]. A spin-glass-like behavior in ferromagnetic phase of CCS is reported by Kitani et al. [8]. The occurrence of a multiferroic state in various spinel systems, including CCS [2] [3] [9] [10], $\mathrm{FeCr}_{2} \mathrm{~S}_{4}$ (FCS) [11], $\mathrm{CdCr}_{2} \mathrm{Se}_{4}$ (CCSe) [10] and $\mathrm{HgCr}_{2} \mathrm{~S}_{4}$ (HCS) [4] is reported. In $\mathrm{CdCr}_{2} \mathrm{~S}_{4}$ (CCS) the ferromagnetic and ferroelectric transitions were found at 84 and $57 \mathrm{~K}$, respectively. An evident anomaly in the dielectric constant at $T_{C}=84$ $\mathrm{K}$ has been reported [2] [3], indicating the magnetoelectric coupling between magnetism and dielectric properties. Magnetic and electric field dependent dielectric and magnetic properties are studied in multiferroic CCS by Sun et al. [12]. Concerning the microscopic origin of the polar moments in CCS and CCSe it can be assumed that the ferroelectric distortions result from an off-center position of the $\mathrm{Cr}^{3+}$-ions, which generates a locally polar but macroscopically isotropic cluster state [13]. The results of Grimes et al. [13] revealed that in CCS and CCSe canonical ferromagnetism coexists at sizable ordering temperatures with a relaxor-ferroelectric state, characterized by a significant relaxational behavior. Both order parameters are strongly coupled. A scenario in which the relaxation mechanism interacts with magnetic order via exchange striction can be considered the most plausible.

There are not so many theoretical investigations of the properties of magnetic relaxor ferroelectrics. Fennie and Rabe [14] have studied the dielectric properties of the ferromagnetic spinel CCS from first principles. The observed results suggested that the observed anomalous dielectric behavior in CCS is not due to the softening of a polar mode. Oliveira et al. [7] have used the Landau theory to demonstrate that a linear coupling between the magnetic and polar order parameters $(\alpha P M)$ is sufficient to justify the appearance of magnetic cluster in the paramagnetic phase of CCS. Zhou et al. [15], based on the spherical random-bond-random-field model and the Heisenberg model, considering the coupling interaction between the relaxor ferroelectricity and magnetism, have calculated the third-order static nonlinear dielectric susceptibility as well as the scaled nonlinear susceptibility of magnetic relaxor ferroelectrics. The important role of the fluctuations of the spin-pair correlations in the change of the dielectric susceptibility around the magnetic phase transition temperature is shown by Xia and Jiang [16] [17] based on the spherical random-bond-random-field (SRBRF) model, the Heisenberg model and a magnetoelectric coupling term. Using the same model Cao et al. [18] have investigated the influence of Fe substitution on the magnetic and static nonlinear dielectric response in the doped magnetic relaxor ferroelectrics CCS. Band structure calculations for $\mathrm{ACr}_{2} \mathrm{X}_{4}(\mathrm{~A}=$ Zn, Cd, Hg and X = O, S, Se) spinels are presented by Yaresko [19].

The aim of the present paper is to study the effect of an applied static magnetic field on the dielectric properties of magnetic relaxor ferroelectric CCS using a microscopic model and the replica method as well as the Green's function theory. 


\section{The Model}

Pioneering studies of magnetic semiconductors in the 1960s showed the coexistence of semiconduction and magnetism in some ferromagnetic semiconductors, such as europium chalcogenides and the spinel compounds CCS and CCSe [20]. But in the last years they are considered as materials as magnetic relaxor ferroelectrics. The Hamiltonian which can describe their magnetic and relaxor ferroelectric properties is:

$$
H=H_{m}+H_{f}+H_{m f} .
$$

$H_{m}$ is the s-d Hamiltonian:

$$
H_{m}=H_{s}+H_{e}+H_{I} .
$$

$H_{s}$ is the Heisenberg Hamiltonian for the ferromagnetically ordered $d$ electrons,

$$
H_{s}=-\frac{1}{2} \sum_{i, j} J_{i j} \mathbf{S}_{i} \cdot \mathbf{S}_{j}-\sum_{i} \mathbf{h} \cdot \mathbf{S}_{i}+H_{s p},
$$

where $\mathbf{S}_{i}$ is the Heisenberg operator of $\mathrm{Cr}^{3+}$ at the lattice site $i$. Ferromagnetic order in CCSe and in CCS appears below $T_{C m}=130 \mathrm{~K}$ and $84 \mathrm{~K}$, respectively, as a result of competition between the direct $\mathrm{Cr}-\mathrm{Cr}$ spin coupling and the near neighbour $\mathrm{Cr}-\mathrm{Se}$ (or S)-Cr exchange interactions. The exchange interaction $J_{i j}$ is a function of the lattice constant and stands for the nearest neighbors $J_{1}>0$ and next nearest neighbors $J_{2}<0$. The frustration plays an important role in these materials. $\mathbf{h}$ is an applied magnetic field.

Strong spin-phonon interaction is reported in CCS [12] [21] [22] [23]:

$$
H^{s p}=-\frac{1}{2} \sum_{i, j} F(i, j) Q_{i} S_{j}^{z}-\frac{1}{4} \sum_{i, j, r} R(i, j, r) Q_{i} Q_{j} S_{r}^{z}+\text { h.c. }
$$

$Q_{i}$ is the normal coordinate of the lattice mode. $F$ and $R$ designate the amplitudes for coupling of phonons to the spin excitations in first and second order, respectively. h.c. represents the hermitian conjugate.

$H_{e}$ is the usual Hamiltonian of the conduction band electrons,

$$
H_{e}=\sum_{\mathbf{q}, \sigma}\left(\epsilon_{\mathbf{q}, \sigma}-\mu\right) a_{\mathbf{q} \sigma}^{+} a_{\mathbf{q} \sigma},
$$

where $a^{+}$and $a$ are the Fermi creation and annihilation operators, $\mu$ is the chemical potential, and $\epsilon_{\mathbf{q}, \sigma}$ are the Bloch energies.

$H_{I}$ couples the two subsystems (3) and (4) by an intraatomic exchange interaction $I$

$$
H_{I}=-\frac{I}{2 N} \sum_{\mathbf{q}, \mathbf{p}}\left[S_{\mathbf{q}-\mathbf{p}}^{+} a_{\mathbf{p}-}^{+} a_{\mathbf{q}^{+}}+S_{\mathbf{q}-\mathbf{p}}^{-} a_{\mathbf{p}+}^{+} a_{\mathbf{q}^{-}}+S_{\mathbf{q}-\mathbf{p}}^{z}\left(a_{\mathbf{p}+}^{+} a_{\mathbf{q}^{+}}-a_{\mathbf{p}-}^{+} a_{\mathbf{q}^{-}}\right)\right] .
$$

The Hamiltonian of the relaxor ferroelectric subsystem $H_{f}$ in the presence of an electric field can be written as [24]

$$
H_{f}=-\frac{1}{2} \sum_{(k, l)} A_{k l} \bar{\sigma}_{k} \cdot \bar{\sigma}_{l}-\sum_{k} \tilde{\mathbf{h}}_{k} \cdot \bar{\sigma}_{k}-\sum_{k} \mathbf{E} \cdot \bar{\sigma}_{k} .
$$


$(k, l)$ means the summation over each distinct pair once, and $\sigma_{k}$ is a dimensionless order parameter field. $A_{k l}$ and $\tilde{\mathbf{h}}_{k}$ are randomly frustrated bonds and random fields, respectively, which are satisfied with a Gaussian distribution. The last are characterized by the variance $\Delta . A_{k l}$ is specified by its random average value $A_{0}$ and root-mean-square variance $A^{2}$. $\mathbf{E}$ is an applied electric field. A ferroelectric ordering in CCS occurs near $T_{C f}=56 \mathrm{~K}$.

There are four order parameters-the localized-spin magnetization $M$ for arbitrary spin $S$, the conduction-electron magnetization $\rho$, the polarization $P$ and the spin glass order parameter $q$ which are given by:

$$
\begin{gathered}
M=\left\langle S^{z}\right\rangle=\frac{1}{N^{2}} \sum_{i, j}\left[(S+0.5) \operatorname{coth}\left[(S+0.5) \beta E_{i j}\right]-0.5 \operatorname{coth}\left(0.5 \beta E_{i j}\right)\right], \\
\rho=\frac{1}{2 N} \sum_{\mathbf{q}, \sigma} \sigma\left\langle a_{\mathbf{q} \sigma}^{+} a_{\mathbf{q} \sigma}\right\rangle, \quad \sigma= \pm 1 \\
P=\beta(1-q)\left(A_{0 e f f} P+E\right), \\
q=\beta^{2} A_{e f f}^{2}\left(q+\Delta / A_{\text {eff }}^{2}\right)(1-q)^{2}+P^{2},
\end{gathered}
$$

with the renormalized exchange interaction constants:

$$
\begin{gathered}
A_{0 e f f}=A_{0}-2 g\left\langle\mathbf{S}_{i} \cdot \mathbf{S}_{j}\right\rangle, \\
A_{e f f}^{2}=A^{2}-4 g\left\langle\mathbf{S}_{i} \cdot \mathbf{S}_{j}\right\rangle+4 g^{2}\left\langle\mathbf{S}_{i} \cdot \mathbf{S}_{j}\right\rangle^{2} .
\end{gathered}
$$

The magnetoelectric coupling term between the magnetic (3) and electric (7) subsystems in the magnetic relaxor ferroelectric CCS and CCSe is proposed to be linear in the electric order parameter and quadratic in the magnetic order parameter:

$$
H_{m f}=g \sum_{i j k} \bar{\sigma}_{i} \mathbf{S}_{j} \cdot \mathbf{S}_{k},
$$

where $g$ is the magnetoelectric coupling constant. It must be noted that Xia and Jiang [16] [17] have chosen a biquadratic magnetoelectric coupling-quadratic in the electric and quadratic in the magnetic order parameters. But this coupling is suitable for multiferroics with very different phase transition temperatures, $T_{C f} \gg T_{C m}$, for example in $\mathrm{BiFeO}_{3}$ or hexagonal $\mathrm{RMnO}_{3}$. But this is here not the case, because both temperatures are close to another, $T_{C f} \leq T_{C m}$ [2], similarly to $\mathrm{RMn}_{2} \mathrm{O}_{5}$, which corresponds to a linear magnetoelectric coupling.

The correlation functions are calculated using the Green's functions $G_{i j}=\left\langle S_{i}^{+} ; S_{j}^{-}\right\rangle$and $g_{i j}=\left\langle a_{i}^{+} ; a_{j}\right\rangle$ All quantities are renormalized due to the magnetoelectric and spin-phonon coupling and must be calculated selfconsistently.

\section{Numerical Results and Discussion}

The temperature dependence of the magnetization and polarization are numerically calculated taking parameters appropriated for $\mathrm{CdCr}_{2} \mathrm{~S}_{4}: J_{1}=0.1 \mathrm{eV}, J_{2}=$ $-0.05 \mathrm{eV}, I=0.3 \mathrm{eV}$ [25], $S=3 / 2, F=4 \mathrm{~cm}^{-1}, R=-0.35 \mathrm{~cm}^{-1}, T_{C m}=84 \mathrm{~K}, T_{C f}=$ 
$56 \mathrm{~K}, A=0.1 \mathrm{eV}, g=0.3 \mathrm{eV}$.

The spin-phonon interaction taken up to second order and the magnetoelectric coupling $g$ renormalize the exchange interaction constant $J_{1}$ to $J_{1}^{\text {eff }}$ which is now temperature dependent:

$$
J_{1}^{e f f}=J_{1}+\frac{2 F^{2}}{\omega_{0}-M R}+2 g\langle\tilde{\sigma}\rangle,
$$

where $M$ is the magnetization and $\omega_{0}$ is the unrenormalized phonon energy.

By the calculation of the remanent polarization $P_{r}(T)$ we have included an electric field of $E=100 \mathrm{kV} / \mathrm{m}$ because there is no ferroelectric transition except in applied electric fields what is typical for relaxor ferroelectrics [26]. The results of $M$ and $P_{r}$ are presented in Figure 1 and Figure 2, respectively. They show a coexistence of ferromagnetism and relaxor ferroelectricity together below nearly $T=56 \mathrm{~K}$, i.e. below the ferroelectric phase transition temperature $T_{C f}$ There is a

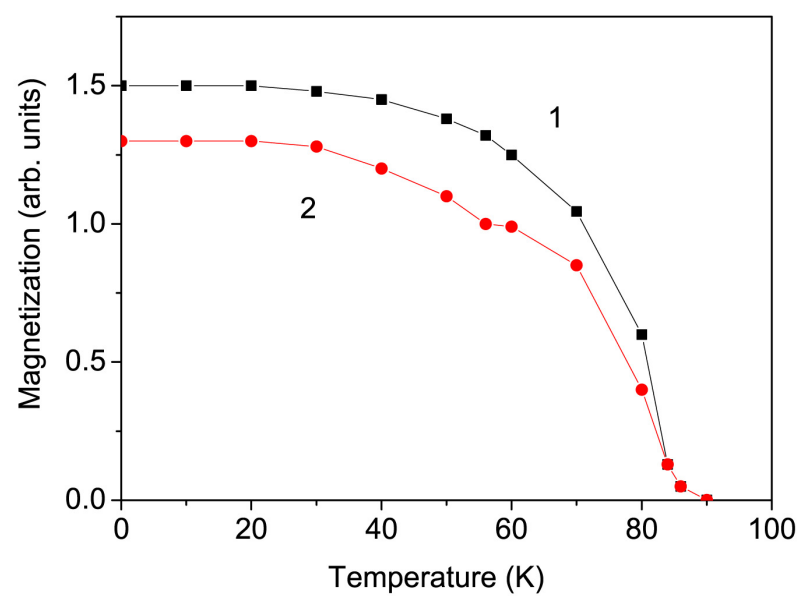

Figure 1. Temperature dependence of the magnetization $M$ in CCS for 500 Oe and different electric field values: $E=0$ (1), $100 \mathrm{kV} / \mathrm{m}(2)$.

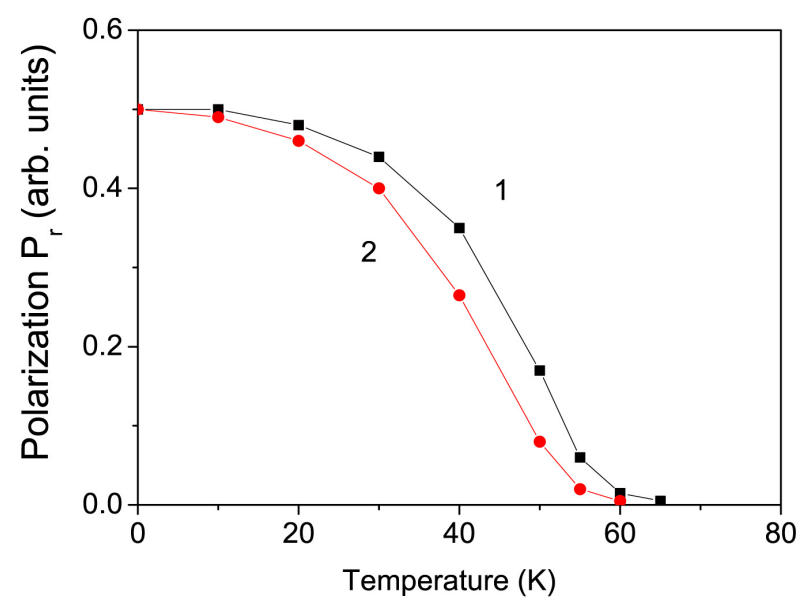

Figure 2. (Color online) Temperature dependence of the remanent polarization $P_{r}$ in CCS for $E=100 \mathrm{kV} / \mathrm{m}$ and different magnetic field values: $h=0$ (1), 500 Oe (2). 
diffuse ferroelectric phase transition, which is characteristic for relaxor ferroelectrics. We have studied also the magnetic field dependence of the remanent polarization. $P_{r}$ and $T_{C f}$ decrease with increasing magnetic field $h$ (Figure 2, curve 2) what is evidence for a multiferroic behavior. The observed results are in good qualitative agreement with the experimental data of Hemberger et al. [2].

A strong decrease in the magnetization $M$ is observed well below $T_{C m}=85 \mathrm{~K}$ with increasing the external electric field $E$ (Figure 1, curve 2). There is a kink around the ferroelectric phase transition $T_{C f}$ The kink increases with increasing $E$ in agreement with the experimental data of Sun et al. [12]. This anomaly is due to the magnetoelectric coupling $g$. It is evidence for the strong correlation among magnetic spin, electric dipole and the lattice in the multiferroic CCS. It must be noted that with increasing spin-phonon interaction $R$ the kink in the temperature dependence of $M$ is deeper and clearer. This shows the importance of the anharmonic spin-phonon interaction and that it must be taken into account in order to obtain correct results. We have observed a similar kink in the temperature dependence of the magnetization in the multiferroic $\mathrm{RMn}_{2} \mathrm{O}_{5}$ [27], where is also valid $T_{C f}<T_{C m}$.

We have calculated also the dielectric function $\epsilon$ from the expression [28]:

$$
\left[\left(\frac{\Lambda}{\epsilon(\mathbf{k}, E)-1}\right)_{\alpha \beta}+\Lambda \frac{k_{\alpha} k_{\beta}}{k^{2}}\right] \tilde{G}^{\beta \gamma}(\mathbf{k}, E)=\delta_{\alpha \gamma} ; \quad \Lambda=4 \pi Z^{2} / v,
$$

where $Z$ is the electron charge and $v$ is the volume, $\tilde{G}$ is the ferroelectric Green's function.

The temperature and magnetic field dependence of the real part of $\epsilon$ is shown in Figure 3. The dielectric function $\epsilon^{\prime}$ of CCS becomes strongly enhanced in the region below the ferromagnetic transition temperature $T_{C m}=84 \mathrm{~K}$ which is strongly correlated with the ferromagnetic ordering. Exchange striction [2] [21], which mechanically induces the strain, is associated with the ferromagnetic

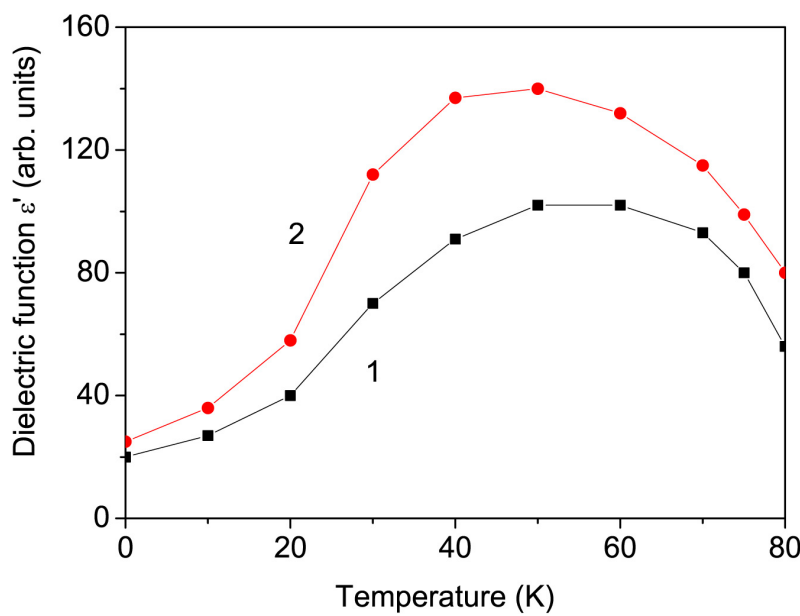

Figure 3. (Color online) Temperature dependence of the real part of the dielectric function $\epsilon^{\prime}$ for $E=100 \mathrm{kV} / \mathrm{m}$ and different magnetic field values: $h=0$ (1), 500 Oe (2). 
ordering around $T_{C m}$ and local lattice distortion takes place inducing the enhancement of dielectric constant. It goes through a broad maximum, which indicates a diffuse phase transition. This broad peak of $\epsilon^{\prime}(T)$ is typical for a relaxor ferroelectric. The observed behavior is in accordance with the experimental data for CCS [2]. The low temperature peak is due to magnetic ordering, which shows that the magnetic and electric orders are coupled. All these characteristics are signatures of the relaxor state of magnetic relaxor ferroelectircs, in agreement with previous reports [4] [10].

The ferroelectric properties strongly depend on the external magnetic field $h$ in multiferroics. Therefore, we have calculated also the magnetic field dependence of $\epsilon^{\prime}$ in CCS, see Figure 3, curve 2. The dielectric function $\epsilon^{\prime}$ increases with increasing $h$ in agreement with the experimental data of Hemberger et al. [2] and Sun et al. [12]. The maximum is shifted to smaller temperature values. A similar shift of $\epsilon^{\prime}$ is reported by Sun et al. [12]. It must be noted that Hemberger et al. [2] and Krohns et al. [9] also observed an increase of the dielectric function $\epsilon^{\prime}$ with increasing the magnetic field and nonzero frequencies in CCS, but the peak shifts to higher temperature values. It must be noted that our calculations are for zero frequencies. In HCS Weber et al. [4] demonstrated also the strong increase of the dielectric constant in external magnetic fields.

\section{Conclusions}

The temperature and magnetic field dependence of relaxor characteristics such as remanent polarization $P_{r}$ and real part of the dielectric function $\epsilon^{\prime}$ are investigated theoretically for CCS. The relaxor polarization and the magnetization appear together below $T \sim 56 \mathrm{~K}$, i.e. below the ferroelectric phase transition temperature $T_{C f}$ A diffuse ferroelectric phase transition and a broad peak for $\epsilon^{\prime}$ are observed which are typical for relaxor ferroelectrics, such as CCS. The polarization decreases whereas the dielectric function increases and the maximum of $\epsilon^{\prime}$ shifts to higher temperature values with increasing the magnetic field $h$. It is shown that CCS is a multiferroic and that the relaxor behavior of magnetic relaxor ferroelectrics such as CCS can be controlled by the application of an external magnetic field.

The magnetization $M$ decreases with increasing electric field $E$. Moreover, there is a kink around the ferroelectric transition temperature $T_{C f}=56 \mathrm{~K}$. The kink is stronger for higher electric fields $E$ and anharmonic spin-phonon interaction constants $R$.

It would be of interest to investigate the influence of cation substitution on the magnetic and dielectric properties in the doped magnetic relaxor ferroelectric CCS. This will be made in a next paper.

\section{Conflicts of Interest}

The authors declare no conflicts of interest regarding the publication of this paper. 


\section{References}

[1] Wei, Y.-X., Jin, C.-Q. and Zeng, Y.-M. (2017) Progress of Relaxor Multiferroic Materials. Journal of Inorganic Materials, 32, 1009-1017. https://doi.org/10.15541/jim20160644

[2] Hemberger, J., Lunkenheimer, P., Fichtl, R., Krug von Nidda, H.-A., Tsurkan, V. and Loidl, A. (2005) Relaxor Ferroelectricity and Colossal Magnetocapacitive Coupling in Ferromagnetic $\mathrm{CdCr}_{2} \mathrm{~S}_{4}$. Nature (London), 434, 364-367.

https://doi.org/10.1038/nature03348

[3] Lunkenheimer, P., Fichtl, R., Hemberger, J., Tsurkan, V. and Loidl, A. (2005) Relaxation Dynamics and Colossal Magnetocapacitive Effect in $\mathrm{CdCr}_{2} \mathrm{~S}_{4}$. Physical Review B, 72, 060103. https://doi.org/10.1103/PhysRevB.72.060103

[4] Weber, S., Lunkenheimer, P., Fichtl, R., Hemberger, J., Tsurkan, V. and Loidl, A. (2006) Colossal Magnetocapacitance and Colossal Magnetoresistance in $\mathrm{HgCr}_{2} \mathrm{~S}_{4}$. Physical Review Letters, 96, 157202. https://doi.org/10.1103/PhysRevLett.96.157202

[5] Hartmann, O., Kalvius, G.M., Waeppling, R., Guenther, A., Tsurkan, V., Krimme, A. and Loidl, A. (2013) Magnetic Properties of the Multiferroic Chromium Thio-Spinels $\mathrm{CdCr}_{2} \mathrm{~S}_{4}$ and $\mathrm{HgCr}_{2} \mathrm{~S}_{4}$. The European Physical Journal B, 86, 148-155. https://doi.org/10.1140/epjb/e2013-31094-4

[6] Tsurkan, V., Hemberger, J., Krimmel, A., Krug von Nidda, H.-A., Lunkenheimer, P., Weber, S., Zestrea, V. and Loidl, A. (2006) Experimental Evidence for Competition between Antiferromagnetic and Ferromagnetic Correlations in $\mathrm{HgCr}_{2} \mathrm{~S}_{4}$. Physical Review B, 73, 224442. https://doi.org/10.1103/PhysRevB.73.224442

[7] Oliveira, G.N.P., Pereira, A.M., Lopes, A.M.L., Amaral,J. S., dos Santos, A.M., Ren, Y., Mendonc, T.M., Sousa, C.T., Amaral, V.S., Correia, J.G. and Araujo, J.P. (2012) Dynamic Off-Centering of $\mathrm{Cr}^{3+}$ Ions and Short-Range Magneto-Electric Clusters in $\mathrm{CdCr}_{2} \mathrm{~S}_{4}$. Physical Review B, 86, 224418. https://doi.org/10.1103/PhysRevB.86.224418

[8] Kitani, S., Tachibana, M. and Kawaji, H. (2014) Spin-Glass-Like Behavior in Ferromagnetic Phase of $\mathrm{CdCr}_{2} \mathrm{~S}_{4}$. Solid State Communications, 179, 16-19.

https://doi.org/10.1016/j.ssc.2013.06.004

[9] Krohns, S., Schrettle, F., Lunkenheimer, P., Tsurkan, V. and Loidl, A. (2008) Colossal Magneto Capacitive Effect in Differently Synthesized and Doped $\mathrm{CdCr}_{2} \mathrm{~S}_{4}$. Physica B, 403, 4224-4227. https://doi.org/10.1016/j.physb.2008.09.024

[10] Hemberger, J., Lunkenheimer, P., Fichtl, R., Weber, S., Tsurkan, V. and Loidl, A. (2006) Multiferroic Behavior in $\mathrm{CdCr}_{2} \mathrm{X}_{4}(\mathrm{X}=\mathrm{S}$; Se). Physica B, 378-380, 363-366. https://doi.org/10.1016/j.physb.2006.01.407

[11] Bertinshaw, J., Ulrich, C., Guenther, A., Schrettle, F., Wohlauer, M., Krohns, S., Reehuis, M., Studer, A.J., Avdeev, M., Quach, D.V., Groza, J.R., Tsurkan, V., Loidl, A. and Deisenhofer, J. (2014) $\mathrm{FeCr}_{2} \mathrm{~S}_{4}$ in Magnetic Fields: Possible Evidence for a Multiferroic Ground State. Scientific Reports, 4, 6079-6087. https://doi.org/10.1038/srep06079

[12] Sun, C.P., Lin, C.C., Her, J.L., Ho, C.J., Taran, S., Berger, H., Chaudhuri, B.K. and Yang, H.D. (2009) Colossal Electroresistance and Colossal Magnetoresistance in Spinel Multiferroic $\mathrm{CdCr}_{2} \mathrm{~S}_{4}$. Physical Review B, 79, 214116. http://apl.aip.org/apl/copyright.jsp

[13] Grimes, N.W. (1972) "Off-Centre" Ions in Compounds with Spinel Structure. The Philosophical Magazine, 26, 1217-1226. https://doi.org/10.1080/14786437208227375

[14] Fennie, C.J. and Rabe, K.M. (2005) Polar Phonons and Intrinsic Dielectric Response 
of the Ferromagnetic Insulating Spinel $\mathrm{CdCr}_{2} \mathrm{~S}_{4}$ from First Principles. Physical Review $B, 72,214123$. https://doi.org/10.1103/PhysRevB.72.214123

[15] Zhou, Q., Cao, H.-X. and Jiang, Q. (2008) The Magnetic Effect on Static Nonlinear Dielectric Response in Magnetic Relaxor Ferroelectrics. Journal of Applied Physics, 104, 093517. https://doi.org/10.1063/1.3008039

[16] Xia, G.S. and Jiang, Q. (2006) Role of the Spin-Pair Correlation in Magnetic Relaxor Ferroelectrics. Journal of Applied Physics, 100, Article ID: 124107. https://doi.org/10.1063/1.2401646

[17] Xia, G.S. and Jiang, Q. (2007) Spin-Pair Correlation Dependent Dynamic Properties in Magnetic Relaxor Ferroelectrics. Journal of Applied Physics, 101, Article ID: 104121. https://doi.org/10.1063/1.2729478

[18] Cao, H.-X., Jiang, Q. and Li, Z.-Y. (2010) Magnetic and Static Nonlinear Dielectric Response in Doped $\mathrm{CdCr}_{2} \mathrm{~S}_{4}$. Ferroelectrics, 409, 190-195. https://doi.org/10.1080/00150193.2010.486240

[19] Yaresko, A.N. (2008) Electronic Band Structure and Exchange Coupling Constants in $\mathrm{ACr}_{2} \mathrm{X}_{4}$ Spinels $(\mathrm{A}=\mathrm{Zn}, \mathrm{Cd}, \mathrm{Hg}$ and $\mathrm{X}=\mathrm{O}, \mathrm{S}, \mathrm{Se})$. Physical Review B, 77, Article ID: 115106. https://doi.org/10.1103/PhysRevB.77.115106

[20] Nagaev, E.I. (1974) Spin Polaron Theory for Magnetic Semiconductors with Narrow Bands. Physica Status Solidi B, 66, 11-60. https://doi.org/10.1002/pssb.2220650102

[21] Rudolf, T., Kant, C., Mayr, F., Hemberger, J., Tsurkan, V. and Loidl, A. (2007) Polar Phonons and Spin-Phonon Coupling in $\mathrm{HgCr}_{2} \mathrm{~S}_{4}$ and $\mathrm{CdCr}_{2} \mathrm{~S}_{4}$ Studied with Far-Infrared Spectroscopy. Physical Review B, 76, Article ID: 174307. https://doi.org/10.1103/PhysRevB.76.174307

[22] Wesselinowa, J.M. and Apostolov, A.T. (1993) Self-Consistent Theory of Spin-Phonon Interactions in Ferromagnetic Semiconductors. Journal of Physics. Condensed Matter, 5, 3555-3564. https://doi.org/10.1088/0953-8984/5/22/007

[23] Wesselinowa, J.M. and Apostolov, A.T. (1996) Anharmonic Effects in Ferromagnetic Semiconductors. Journal of Physics. Condensed Matter, 8, 473-488. https://doi.org/10.1088/0953-8984/8/4/012

[24] Pirc, R. and Blinc, R. (1999) Spherical Random-Bond-Random-Field Model of Relaxor Ferroelectrics. Physical Review B, 60, 13470-13478. https://doi.org/10.1103/PhysRevB.60.13470

[25] Haas, C. (1968) Spin-Disorder Scattering and Magnetoresistance of Magnetic Semiconductors. Physical Review, 168, 531-538. https://doi.org/10.1103/PhysRev.168.531

[26] Cowley, R.A., Gvasaliya, S.N., Lushnikov, S.G., Roessli, B. and Rotaru, G.M. (2011) Relaxing with Relaxors: A Review of Relaxor Ferroelectrics. Advances in Physics, 60, 229-327. https://doi.org/10.1080/00018732.2011.555385

[27] Bahoosh, S.G., Wesselinowa, J.M. and Trimper, S. (2013) Microscopic Approach to the Magnetoelectric Effect in $\mathrm{RMn}_{2} \mathrm{O}_{5}$. Physica Status Solidi B, 250, 1816-1824. https://doi.org/10.1002/pssb.201248378

[28] Vaks, V.G. (1973) Introduction to the Microscopic Theory of Ferroelectrics. Nauka, Moscow, 158. 